\title{
Predicting the Pullout Capacity of Small Ground Anchors Using Nonlinear Integrated Computing Techniques
}

\author{
Mosbeh R. Kaloop, ${ }^{1,2,3}$ Jong Wan Hu, ${ }^{1,2}$ and Emad Elbeltagi ${ }^{4,5}$ \\ ${ }^{1}$ Department of Civil and Environmental Engineering, Incheon National University, Incheon, Republic of Korea \\ ${ }^{2}$ Incheon Disaster Prevention Research Center, Incheon National University, Incheon, Republic of Korea \\ ${ }^{3}$ Department of Public Works and Civil Engineering, Mansoura University, Mansoura, Egypt \\ ${ }^{4}$ Department of Structural Engineering, Mansoura University, Mansoura, Egypt \\ ${ }^{5}$ Department of Civil Engineering, Mansoura Higher Institute for Engineering and Technology, Talkha, Egypt
}

Correspondence should be addressed to Jong Wan Hu; jongp24@incheon.ac.kr

Received 6 January 2017; Accepted 29 March 2017; Published 19 April 2017

Academic Editor: Paulo B. Gonçalves

Copyright (C) 2017 Mosbeh R. Kaloop et al. This is an open access article distributed under the Creative Commons Attribution License, which permits unrestricted use, distribution, and reproduction in any medium, provided the original work is properly cited.

\begin{abstract}
This study investigates predicting the pullout capacity of small ground anchors using nonlinear computing techniques. The inputoutput prediction model for the nonlinear Hammerstein-Wiener (NHW) and delay inputs for the adaptive neurofuzzy inference system (DANFIS) are developed and utilized to predict the pullout capacity. The results of the developed models are compared with previous studies that used artificial neural networks and least square support vector machine techniques for the same case study. The in situ data collection and statistical performances are used to evaluate the models performance. Results show that the developed models enhance the precision of predicting the pullout capacity when compared with previous studies. Also, the DANFIS model performance is proven to be better than other models used to detect the pullout capacity of ground anchors.
\end{abstract}

\section{Introduction}

Light structures, which are built in open areas, are supported with the ground using small anchors. Such anchors are designed to resist tensile and uplift forces [1-4] and are usually supported at a shallow depth (about $1 \mathrm{~m}$ ) with small pullout capacity $[2,5,6]$. Therefore, designers rarely put efforts into designing such small ground anchors [5]. In contrast, Shahin and Jaksa [7] introduced new design criteria for small anchors based on advanced prediction models.

The numerical prediction models are used to detect the pullout capacity of small ground anchors based on inputoutput mapping for the in situ data. Shahin and Jaksa [7] utilized 119 anchors' test data to introduce prediction models. They used the neural networks technique to extract the pullout capacity [7]. In addition, Shahin and Jaksa [2, 6] used artificial neural network (ANN) model for the design of small anchors and they were able to predict the pullout capacity. Samui et al. [5] developed a prediction model based on the least square support vector machine (LSSVM) to detect the pullout capacity of small anchors; and they concluded that the LSSVM performs better than the ANN [5].

Nowadays, integrated system identifications are used to design nonlinear input-output prediction models $[8,9]$. In general, these models can be divided into multi-input multioutput (MIMO), single-input single-output (SISO), or multiinput single-output (MISO). The selection of the appropriate model depends on the collected data and sensitivity of the input and output variables. Most common integrated identification models are presented in [8] and it is reported that the Hammerstein-Wiener model outperformed other models [8]. Also, it is found that the nonlinear HammersteinWiener model performance is better than the linear one [10]. On the other hand, the adaptive neurofuzzy inference system (ANFIS) is used widely for the designing of prediction's models; more details on the ANFIS model design and previous studies can be found in [11-14]. The performance of the ANFIS model is better with MISO variables [13, 14]. Arsava et al. [14] introduced a time delayed-ANFIS (DANFIS) prediction model for the control structures, and 


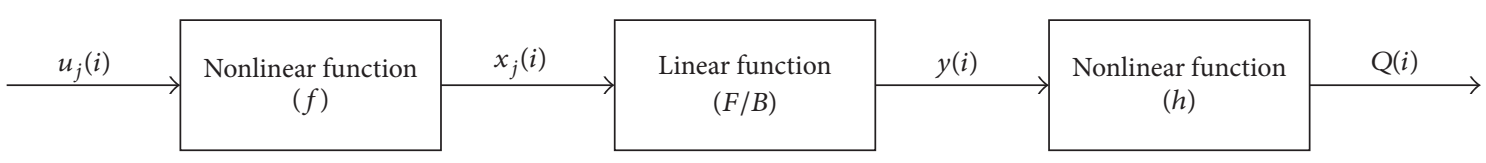

FIgURE 1: MISO-NHW model diagram structure.

they found that DANFIS model performance is much better than conventional ANFIS models. Based on the above review, the nonlinear Hammerstein-Wiener (NHW) and DANFIS models can be used to detect the pullout capacity. Therefore, a new model will be developed to detect the pullout capacity, and the results will be compared with ANN [2] and LSSVM [5] models based on Shahin and Jaksa [2] data collection.

The objectives of this study are the following: (1) to examine the capability of the NHW and DANFIS models for predicting small ground anchors pullout capacity; (2) to compare the performance of developed models with previous studies; and (3) to study the significance of input variables on pullout capacity of small ground anchor.

\section{Material and Methods}

2.1. Prediction Models. The MISO prediction models, NHM and DANFIS, are utilized in this study to extract the pullout capacity of small ground anchors. These models are described in the following subsections.

\subsubsection{Nonlinear Hammerstein-Wiener Model. The NHW} model is an integrated prediction model using nonlinear and linear transforming functions [8]. The model includes input and output nonlinear functions and linear model connected the input and output functions [10]. The nonlinear one-layer sigmoid and wavelet networks, saturation, one-dimension polynomial, and piecewise functions are used for the input $(f)$ and output $(h)$ transforming [15]. In addition, the similar polynomial functions ( $B$ and $F$ ) are defined in the timeshift operator. Figure 1 represents the NHW model diagram. To predict the pullout capacity $(Q(i))$, the input variables $\left(u_{j}(i)\right)$, and transforming results $x_{j}(i)$ and $y(i)$ are utilized and calculated. More details for the NHW model can be found in $[16,17]$.

In this study, four input variables are used to predict the pullout capacity of a MISO model. The trail and errors method is used to select the input and output nonlinearity functions. Therefore, the nonlinearity input function is applied to each input variable $(j)$, and the output $x_{j}(i)$ of each variable can be calculated as follows:

$$
x_{j}(i)=f\left(u_{j}(i)\right) .
$$

The linear output block $y(i)$ is a summation of the inputs as follows:

$$
y(i)=\sum_{j=1}^{n} \frac{B_{j}(k)}{F_{j}(k)} x_{j}(i),
$$

where $n$ is the number of inputs for a MISO model and $B_{j}(k)$ and $F_{j}(k)$ are polynomials defined in the time-shift operator $k$. The model order is chosen based on zero order $\left(B_{j}\right)$ and pole order $\left(F_{j}\right)$, with delays set to zero and $n$ selected as 4 . The zero-pole orders are obtained using the prediction error method. As such, the pullout capacity can be calculated as follows:

$$
Q(i)=h(y(i))
$$

In this paper, the prediction trials were performed with the Matlab command nlhw of the system identification toolbox. Moreover, the models were obtained using model error in which the minimized criterion is the square of the errors, normalized by the length of the data set. In addition, the models performances are evaluated.

2.1.2. Delay Inputs for the Adaptive Neurofuzzy Inference System (DANFIS). The time delayed adaptive neurofuzzy inference system (DANFIS) is proposed in [14] to predict the complex nonlinear behavior of smart structures. In this paper, the DANFIS model is developed to predict the pullout capacity of small ground anchors based on MISO parameters. Figure 2 illustrates the developed model using four input data sets and one delay for the output variable. The ANFIS model consists of a set of fuzzy rules with appropriate membership functions to generate the stipulated input-output pairs in the solution of uncertain and ill-defined systems $[12,14,18,19]$. As presented in Figure 2, the ANFIS model contains five layers that are the input, input membership function (MF), rules list, output MF, and the output layers. Therefore, it is important to define the types and the values of MF for each input variable. Figure 2 shows two MFs for each variable, as shown in the input MF layer.

The process of the ANFIS model can be found in $[14,18]$. As presented in Figure 2, the ANFIS model can be used for mapping the nonlinear MISO variables [20]. In this case, the nonlinear MISO mapping model can be expressed as follows $[14,20]$ :

$$
Q(l)=g\left(u_{1}^{l-d}, u_{2}^{l-d}, \ldots, u_{n}^{l-d}, Q^{l-d}, e^{l-d}\right)+e(l),
$$

where $\left[u_{1}, u_{2}, \ldots, u_{n}\right]$ are the input variables, $Q$ is the model output (pullout capacity), $e$ is the model error, $g$ is a scalar nonlinear mapping function, and the time delay is represented by the term $d$. In this study, four input variables are used and the time delay is assigned a value of one. In general, the if-then rules for the ANFIS model depend on the number of MFs. For each rule, the ANFIS fuzzy model of Takagi and Sugeno (TS) [21] can be applied as follows [18].

Assuming first that $l=2$, while $l=2,3, \ldots, n$; $n$ is the number of measurements; and $d=1$, as presented in Figure 2, the model rule $i$ for the four inputs can be processed as follows. 


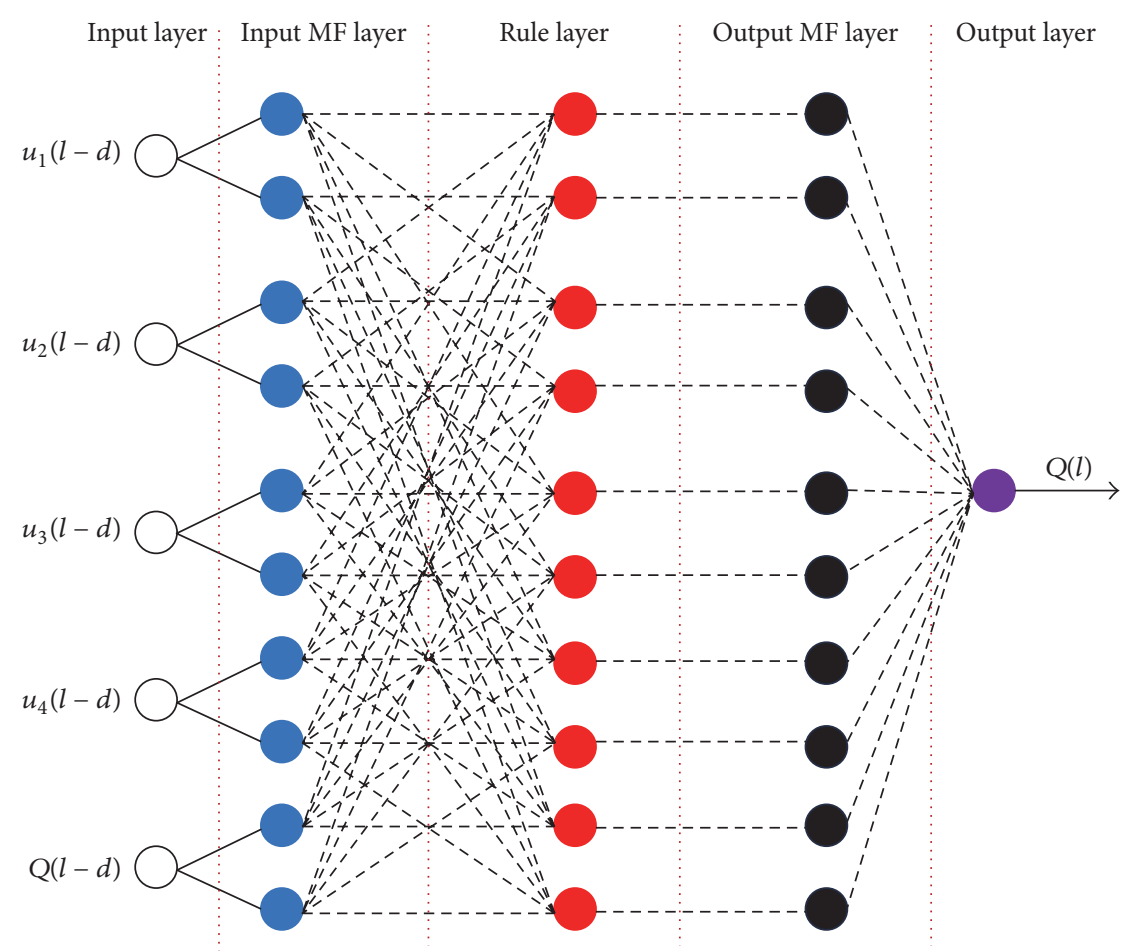

FIGURE 2: MISO-DANFIS model diagram structure.

Rule $i$ is as follows: if $u_{1}$ is $A, u_{2}$ is $B, u_{3}$ is $C, u_{4}$ is $D$, and $Q$ is $F$, then

$$
f_{i}=p_{i} u_{1}+q_{i} u_{2}+k_{i} u_{3}+m_{i} u_{4}+g_{i} Q+r_{i}
$$

where $\left[u_{1}, u_{2}, u_{3}, u_{4}\right]$ are the input variables, $Q$ is the delayed output variable (pullout capacity), $f$ is the output of the TS fuzzy system, and $p_{i}, q_{i}, k_{i}, m_{i}, g_{i}, r_{i}$ are the consequent parameters [18]. Therefore, as shown in Figure 2, the output of the five layers can be presented as follows:

\section{The Output of the Input MF Layer $\left(O_{i}^{1}\right)$}

$$
O_{i}^{1}=\mu_{A_{i}}\left(u_{1}\right), \mu_{B_{i}}\left(u_{2}\right), \mu_{C_{i}}\left(u_{3}\right), \mu_{D_{i}}\left(u_{4}\right), \mu_{F_{i}}(Q),
$$

where $\mu_{A_{i}}, \mu_{B_{i}}, \mu_{C_{i}}, \mu_{D_{i}}$, and $\mu_{F_{i}}$ are the MFs for the input variables of the model. The MF shape is divided into continuous and piecewise differentiable functions with normalized output $\left(\begin{array}{ll}0 & 1\end{array}\right)[12,18]$. Triangular MFs are used which can be presented for the first input $\left(u_{1}\right)$ as follows (the same relation can be found for each input variable):

$$
\mu_{A_{i}}\left(u_{1}\right)=\max \left(\min \left(\frac{u_{1}-a}{b-a}, \frac{c-u_{1}}{c-b}\right), 0\right),
$$

where the parameters $a, b$, and $c$ are the triangular MF values. These parameters can be called the premise parameters as they are the adjustable parameters in the premise part.
The Output of the Rule Layer. This layer has two processes; the first is calculating the firing strength of each fuzzy rule, as follow:

$$
\begin{aligned}
O_{i}^{2}= & w_{i} \\
= & \mu_{A_{i}}\left(u_{1}\right) \times \mu_{B_{i}}\left(u_{2}\right) \times \mu_{C_{i}}\left(u_{3}\right) \times \mu_{D_{i}}\left(u_{4}\right) \\
& \times \mu_{F_{i}}(Q) .
\end{aligned}
$$

The second is normalizing the firing strength, as follows:

$$
O_{i}^{3}=\bar{w}_{i}=\frac{w_{i}}{\sum_{j=1}^{N} w_{j}},
$$

where, $N$ is the number of input variables.

The Output of the Output MF Layer. In this layer, the node functions $\left(f_{i}\right)$ are applied with the previous layer output; the first-order TS model is used and the output of this layer can be expressed as follows:

$$
\mathrm{O}_{i}^{4}=\bar{w}_{i} \times f_{i} .
$$

The Output of the Output Layer. As the last step, the output of this layer is calculated as follows:

$$
O_{i}^{5}=Q(l)=\sum_{i=1}^{N} \bar{w}_{i} \times f_{i} .
$$

Based on (4) and (11), to estimate the $j$ element $(j=i-1)$, the DANFIS output is calculated as follows [14, 22]:

$$
O_{j}^{5}=Q(l)=\sum_{j=1}^{N} \bar{w}_{j} \times f_{j} .
$$



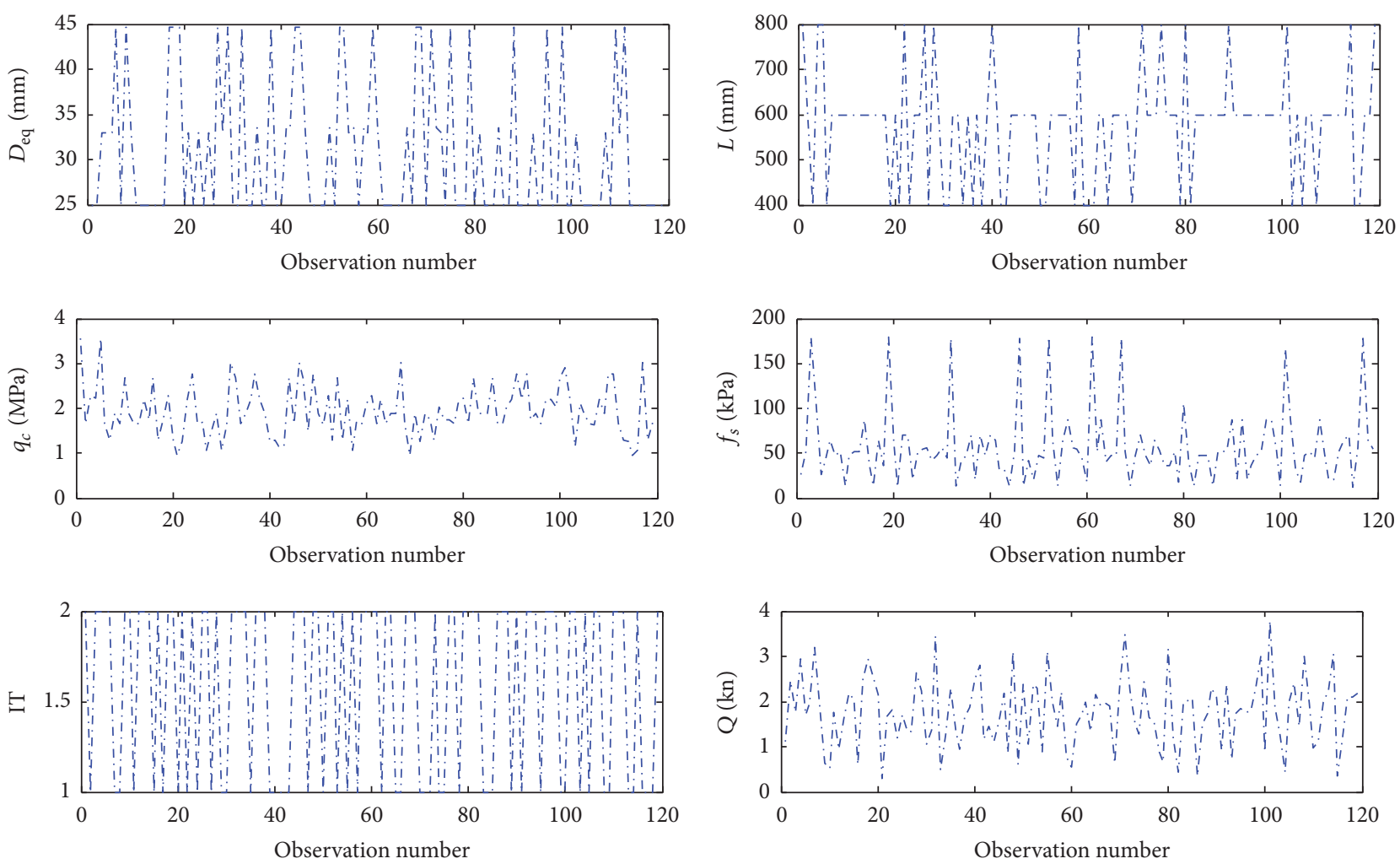

FIgURE 3: Database for field pullout tests (from Samui et al. [5]).

2.2. Case Study. To evaluate the developed models, the field data of 119 anchors are derived using an in situ test database from Shahin and Jaksa [2]. Figure 3 represents the data points and parameters that are considered in this study. As presented in Figure 3, the input variables are the equivalent anchor diameter $\left(D_{\text {eq }}\right)$, embedment depth $(L)$, average cone resistance $\left(q_{c}\right)$ along the embedment depth, average sleeve friction $\left(f_{s}\right)$ along the embedment depth, and installation technique (IT) and the anchor pullout capacity, $(Q)$, is the output. The installation techniques used in this case are static and dynamic cases which are represented by 1 and 2, respectively, as shown in Figure 3. The anchor's types and properties and the anchor's tests process are discussed and presented in [2]. Moreover, the input variables measurements and evaluation, soil properties, and number of tests, as well the monitoring of the anchor pullout capacity, are presented in [7].

The data are divided into training and testing subsets as presented in [5]. The first 83 data points (70\%) are selected as the training dataset and the remaining 36 data points (30\%) are considered as the testing datasets. The statistical analyses (maximum (Max.), minimum (Min.), mean $(M)$, and standard deviation (SD) values) for the training and testing datasets are presented in Table 1.

From Table 1, the statistical measurements for the training and testing datasets show good agreement, meaning that both of them represent almost similar distributions. Before the models simulation, the input and output parameters are normalized by scaling them between 0.2 and 0.8 using (13) to eliminate their dimension effects and to ensure that all variables receive equal attention during training; moreover, it gives the models more flexibility to estimate beyond the training range [23].

$$
x_{\mathrm{eq}}=a_{1}\left(x-x_{\min }\right) \backslash\left(x_{\max }-x_{\min }\right)+a_{2},
$$

where $x_{\min }$ and $x_{\max }$ are minimum and maximum values, respectively; the constant range values $a_{1}$ and $a_{2}$ equal 0.6 and 0.2 , respectively; the equivalent parameter $x_{\mathrm{eq}}$ is scaled between 0.2 and 0.8 .

\subsection{Sensitivity of the Input Variables and Model Performance} Criteria. The data sensitivity is studied based on the previous models designed with the same database $[2,5,7]$. Shahin and Jaksa [7] evaluated the sensitivity of the ANN model with different input variables. Their results show that, during training, the best performance was obtained using the $D_{\text {eq }}$, $L, f_{s}$, and IT input variables, while during validation, the model performed better when sing the $D_{\mathrm{eq}}, L, q_{c}$, and $f_{s}$ input variables. Moreover, Shahin and Jaksa [2] concluded that the ANN model with four input variables, $D_{\text {eq }}, L, f_{s}$, and IT, performed the best, while Samui et al. [5] found that the sensitivity of the $q_{c}$ and $f_{s}$ is higher than that of $D_{\text {eq }}, L$ and the sensitivity of the IT is low. Therefore, because of the inconsistency of the previous studies, the sensitivity of the input variables should be studied first. However, the correlation coefficient between the inputs and output variables is studied first to evaluate the sensitivity of variables, while it can be used to measure the interdependency between successive input and output variables [24]. Second, simple 
TABLE 1: Statistical measurements for the training and testing datasets.

\begin{tabular}{|c|c|c|c|c|c|c|}
\hline Statistical parameters & $D_{\text {eq }}(\mathrm{mm})$ & $L(\mathrm{~mm})$ & $q_{c}(\mathrm{MPa})$ & $f_{s}(\mathrm{kPa})$ & IT & $Q(\mathrm{kN})$ \\
\hline \multicolumn{7}{|c|}{ Training dataset } \\
\hline Max. & 44.60 & 800.00 & 3.55 & 179.71 & 2.00 & 3.47 \\
\hline Min. & 25.00 & 400.00 & 0.95 & 12.22 & 1.00 & 0.29 \\
\hline$M$ & 31.66 & 571.08 & 1.91 & 58.01 & 1.60 & 1.73 \\
\hline SD & 7.88 & 125.46 & 0.58 & 42.18 & 0.49 & 0.77 \\
\hline \multicolumn{7}{|c|}{ Testing dataset } \\
\hline Max. & 44.60 & 800.00 & 3.03 & 178.26 & 2.00 & 3.80 \\
\hline Min. & 25.00 & 400.00 & 0.95 & 12.22 & 1.00 & 0.35 \\
\hline$M$ & 28.85 & 594.44 & 1.98 & 56.62 & 1.56 & 1.80 \\
\hline $\mathrm{SD}$ & 7.00 & 101.26 & 0.55 & 36.70 & 0.50 & 0.78 \\
\hline
\end{tabular}

TABLE 2: Correlation coefficient between input and output variables.

\begin{tabular}{lccccc}
\hline Variables & $D_{\mathrm{eq}}$ & $L$ & $q_{c}$ & $f_{s}$ & IT \\
\hline$Q$ & 0.15 & 0.44 & -0.11 & 0.54 & -0.26 \\
\hline
\end{tabular}

regression model is used to identify the sensitivity of the input variables, as follows:

$$
\mathrm{Q}=a+b_{1} D_{\mathrm{eq}}+b_{2} L+b_{3} q_{c}+b_{4} f_{s}+b_{5} \mathrm{IT},
$$

where, $a, b_{1}, b_{2}, b_{3}, b_{4}, b_{5}$ are the unknown parameters for the regression model. These parameters can be estimated and evaluated using the least square method, as presented in [25, 26]. To examine the significance of each variable, the $t$-test, statistical evaluation, is studied. The $t$ values are compared with predetermined $95 \%$ confidence and $t_{f, 95 \%}$ confidence limit of $t$ distribution; $f$ is the freedom order. The variables within $95 \%$ are considered highly significant to predict the pullout capacity.

In this study, three criteria are used to evaluate the performance of the models design. The first criterion is the correlation coefficient $(R)$, which provide linear dependency information between observation and prediction values. The second statistical criterion is the mean absolute error (MAE), which measures the close prediction values to the eventual outcomes. Finally, the root mean square error (RMSE) is utilized to describe the average magnitude of the errors by giving more weight to large errors.

\section{Results and Discussions}

3.1. Sensitivity Analysis. The scaled data are used in this section to evaluate the variables sensitivity. The correlations between the input and output variables are presented in Table 2.

From Table 2, it can be seen that the degrees of linear dependence between pullout capacity and average sleeve friction and embedment depth are higher than equivalent anchor diameter variables. In addition, the dependencies of the variables on the average cone resistance and installation technique to predict the pullout capacity are low. This indicates that the average sleeve friction, embedment depth, and equivalent anchor diameter variables have more influence on the pullout capacity and this is also reported by Shahin and Jaksa [2].

The simple regression model, as presented in (14), is evaluated and analyzed in Table 3 . Four regression models based on the previous studies, [2, 5-7], are presented to evaluate the sensitivity of the input variables. The models are applied to study the effect of each variable on predicting the pullout capacity, and the correlation coefficients $(R)$ for the prediction models are calculated. The standard deviations of these coefficients are estimated by the least square method. The significance of the estimated coefficients is tested from the zero-expected value in accordance with the $t_{f, 95 \%}$ confidence limit of the $t$ distribution dependent on the $f$ degree of freedom at the $95 \%$ confidence level.

As a result of the models correlation and $t$-test evaluations, the prediction pullout capacity of models 1 and 3 was found to be equally correlated with original pullout capacity. Moreover, it can be seen that the coefficients variables of $D_{\text {eq }}, L$, and $f_{s}$ are significant for the four models, while the coefficients variables of $q_{c}$ and IT are not significant. Hence, the linear trend of $D_{\mathrm{eq}}, L$, and $f_{s}$ variables are high and the prediction effectiveness of the $q_{c}$ variable is higher than the IT variable. Therefore, the sensitivity effects of variables $D_{\text {eq }}$, $L, q_{c}$, and $f_{s}$ in the prediction model are high, and these variables are considered in this study. Herein, it should be mentioned that the sensitivity results in this study are in agreement with Samui et al. [5] and the validation evaluation of the ANN of Shahin and Jaksa [7] for the same case study.

3.2. Models Analysis. Shahin and Jaksa [6] predicted the pullout capacity based on two models and three methods; B-spline neurofuzzy (B-spline-NF) and back-propagation multilayer perceptrons ANN (MLP-ANN) models are used, and the Laboratories Central des Ponts et Chaussees (LCPC) [27], Das [28], and Bowles [29] methods are utilized. In addition, Samui and Sitharam [30] applied the Relevance Vector Machine (RVM) prediction model with different kernels (Gaussian (RVM-G), polynomial (RVM-P), and spline (RVM-spline)). Also, Samui et al. [5] predicted the pullout capacity using least square support vector machine (LSSVM) model. Figure 4 illustrates the $R$ and RMSE values for the previous studies. 
TABLE 3: Linear trend component and coefficient test for the regression models.

\begin{tabular}{|c|c|c|c|c|c|c|c|c|}
\hline & Model & $t_{a}$ & $t_{b 1}$ & $t_{b 2}$ & $t_{b 3}$ & $t_{b 4}$ & $t_{b 5}$ & $R$ \\
\hline 1 & $Q=a+b_{1} D_{\text {eq }}+b_{2} L+b_{3} q_{c}+b_{4} f_{s}+b_{5} \mathrm{IT}$ & 5.5 & 2.5 & 7.6 & -3.8 & 9.8 & -2.8 & 0.77 \\
\hline 2 & $\mathrm{Q}=a+b_{1} D_{\mathrm{eq}}+b_{2} L+b_{4} f_{s}$ & 2.4 & 2.6 & 6.2 & - & 7.5 & - & 0.70 \\
\hline 3 & $Q=a+b_{1} D_{\text {eq }}+b_{2} L+b_{3} q_{c}+b_{4} f_{s}$ & 4.9 & 2.6 & 8.3 & -5.2 & 9.2 & - & 0.77 \\
\hline 4 & $Q=a+b_{1} D_{\mathrm{eq}}+b_{2} L+b_{4} f_{s}+b_{5} \mathrm{IT}$ & 4.1 & 2.6 & 6.1 & - & 9.1 & -4.4 & 0.74 \\
\hline
\end{tabular}

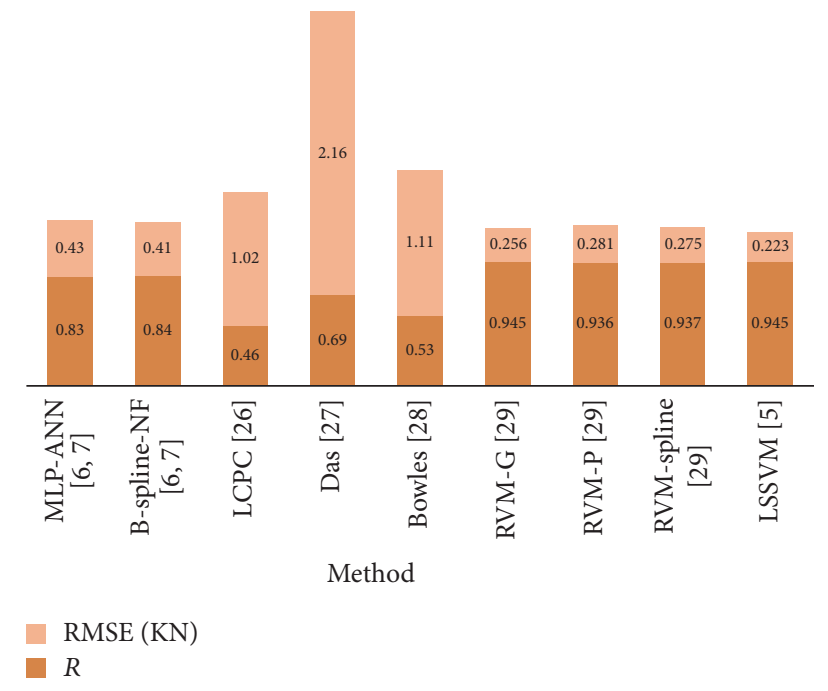

FIgURE 4: Correlation coefficient $(R)$ and RMSE for the predicted pullout capacity of previous studies.

From Figure 4, the vector machine method is the best to detect the pullout capacity of small ground anchors, while the worst case is the Das method. The better method is the LSSVM with high $R=0.945$ and low $\mathrm{RMSE}=0.223$. Samui et al. [5] used all variables to design the model and they found that the sensitivity of $q_{c}$ is higher than $D_{\text {eq }}, L$, and $f_{s}$ variables. Based on Samui et al. [5] and the sensitivity analysis performed in Section 3.1, the current models are designed. In this study, two models are developed, NHW and DANFIS, using $D_{\text {eq }}, L, q_{c}$, and $f_{s}$ as input variables and pullout capacity (Q) as the output variable.

To assess the developed models, the models are programmed on Matlab. In the training phase, 83 datasets are selected and the coefficients of the models have been chosen by trial and error. In the NHW model, the same nonlinear functions for the inputs and output are used. The inputoutput nonlinear sigmoid functions and wavelet networks, saturation, one-dimension polynomial, and piecewise functions are applied with 50 iterations. In addition, the order chosen of linear function $\left(B_{j}\right.$ and $\left.F_{j}\right)$ was $\left[\begin{array}{llll}1 & 1 & 1 & 1\end{array}\right]$ and $\left[\begin{array}{llll}2 & 2 & 8 & 8\end{array}\right]$ with delays set to zero for $D_{\text {eq }}, L, q_{c}$, and $f_{s}$, respectively. This order is selected to compare the functions based on trial-and-error approach. The $R$-values for the sigmoid, wavelet, and piecewise functions are found to be $0.99,0.35$, and 0.60 , respectively. Therefore, the sigmoid function is selected as a nonlinear function for the input and output mapping. The better trials for the linear function orders are presented in Table 4.
TABLE 4: Linear function order trails evaluation.

\begin{tabular}{|c|c|c|}
\hline$\left[\begin{array}{llll}B_{D_{\mathrm{eq}}} & B_{L} & B_{q_{c}} & B_{f_{s}}\end{array}\right]$ & {$\left[\begin{array}{llll}F_{D_{\mathrm{eq}}} & F_{L} & F_{q_{c}} & F_{f_{s}} \\
\end{array}\right.$} & $R$ \\
\hline$\left[\begin{array}{llll}2 & 2 & 2 & 2\end{array}\right]$ & {$\left[\begin{array}{llll}2 & 2 & 2 & 2\end{array}\right]$} & 0.70 \\
\hline$\left[\begin{array}{llll}1 & 1 & 1 & 1\end{array}\right]$ & {$\left[\begin{array}{llll}2 & 2 & 5 & 5\end{array}\right]$} & 0.95 \\
\hline$\left[\begin{array}{llll}1 & 1 & 1 & 1\end{array}\right]$ & {$\left[\begin{array}{llll}2 & 2 & 8 & 8\end{array}\right]$} & 0.99 \\
\hline$\left[\begin{array}{llll}1 & 1 & 1 & 1\end{array}\right]$ & {$\left[\begin{array}{llll}8 & 8 & 2 & 2\end{array}\right]$} & 0.90 \\
\hline
\end{tabular}

The presented values in Table 4 show that the model order of pole is more effective than zeros order; in addition, it is seen that with increased values of the orders for the $q_{c}$ and $f_{s}$ variables, the performance of the model becomes better. That means the sensitivity of $q_{c}$ and $f_{s}$ is higher than that of $D_{\text {eq }}, L$. However, the NHW model contains a sigmoid function for the input and output variables, and $\left[\begin{array}{llll}1 & 1 & 1 & 1\end{array}\right]$ and $\left[\begin{array}{llll}2 & 2 & 8 & 8\end{array}\right]$ orders for the linear function are utilized to predict the pullout capacity.

On the other hand, the DANFIS model is designed using the four input variables and one-time-delayed output; and the pullout capacity is the output value. Two MF functions for each variable are used in this case with 92 nodes and 62 model coefficients. Different MF types are evaluated with 50 iterations, and the best predicted pullout capacity $(R=$ 0.99 ) is obtained using triangular MFs, and this result is reported, also, in Shahin and Jaksa [6, 7]. Figure 5 represents the DANFIS model design.

In this model, 32 fuzzy rules are used, and the numbers of linear and nonlinear coefficients are 32 and 30 , respectively. The application of the model is presented in Figure 5(a) which includes the five basic steps of the calculation. The model begins with the fuzzification of the inputs; then the rules are applied using the fuzzy operation (AND) and the implication and transfer data from premise to consequent. After that, the aggregation of the consequents across rules and output defuzzification are defined to estimate the pullout capacity. The typical model is presented in Figure 5(b), and the adjusted MFs for the five input variables are shown in Figure 5(c).

The performances of the designed NHW and DANFIS models are presented in Figure 6 and Table 5. Figure 6 illustrates the scatter plot of the training dataset, and Table 5 presents the statistical performance and comparison of the developed models and the LSSVM model. From Figure 6, it can be seen that the performance of the DANFIS model is better than the NHW model. The coefficients of the linear fitting for the relation between the observed and predicted 


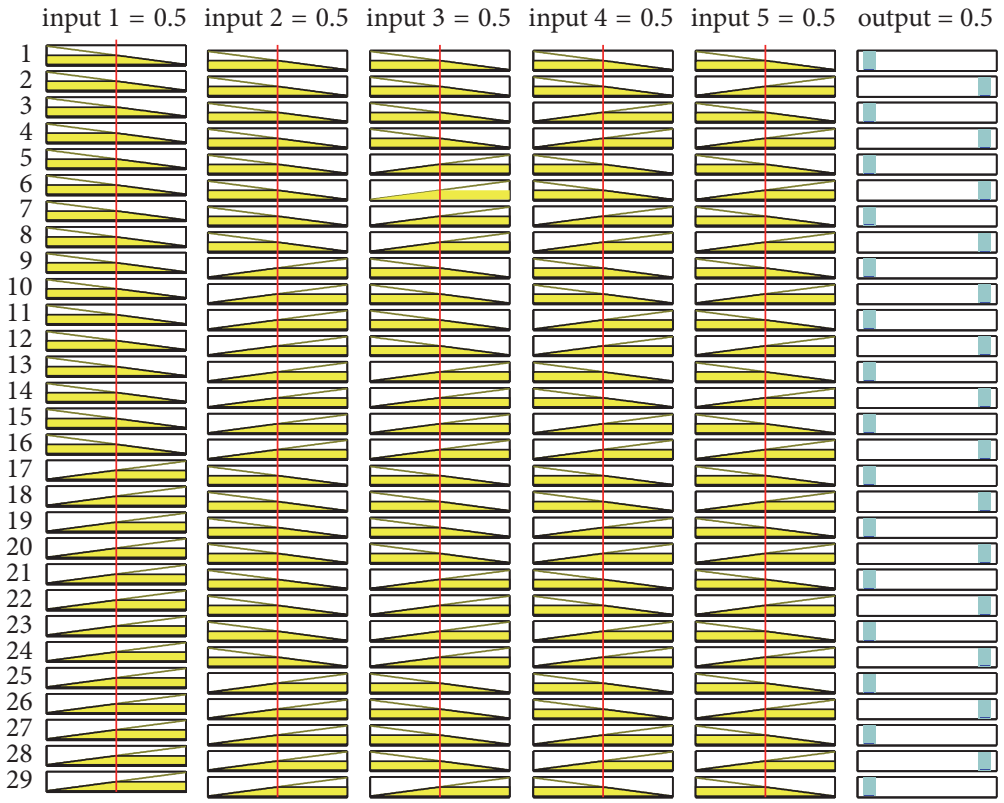

(a)
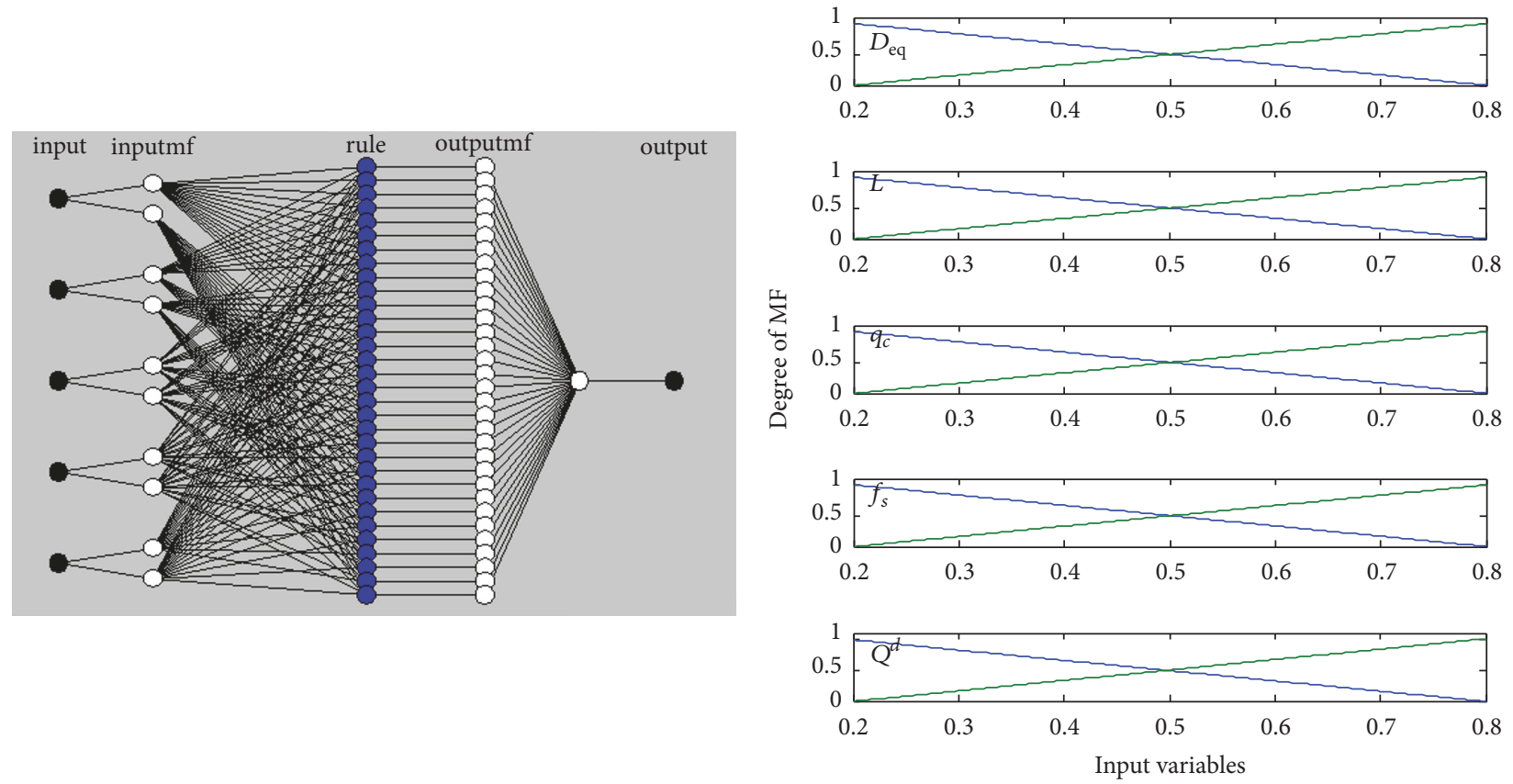

(b)

(c)

FIGURE 5: DANFIS model design: (a) model application, (b) typical model architecture with five inputs, and (c) adjusted MF for the five inputs variables.

pullout capacity of small ground anchors are better with the DANFIS model. In addition, the NHW and the DANFIS models performed better than the LSSVM [5] model. As such, the developed models performances are acceptable to predict the pullout capacity without information losses of the measured values.

The observed and the predicted values of the pullout capacity by the NHW and the DANFIS models are shown in Figure 7 for the testing dataset with high agreement between them. Table 6 shows the statistical performance of the developed models and the LSSVM model for the testing
TABle 5: Comparison between the developed models and the LSSVM [5] model for the training data.

\begin{tabular}{lccc}
\hline Model & RMSE $(\mathrm{KN})$ & MAE $(\mathrm{KN})$ & $R$ \\
\hline LSSVM [5] & 0.22 & 0.19 & 0.94 \\
NHW & $6.65 E-3$ & $8.25 E-3$ & 0.99 \\
DANFIS & $3.91 E-6$ & $2.40 E-6$ & 0.99 \\
\hline
\end{tabular}

dataset. The LSSVM model outperforms the ANN model results for the testing dataset; the MAE is 0.31 and $0.21 \mathrm{KN}$, 


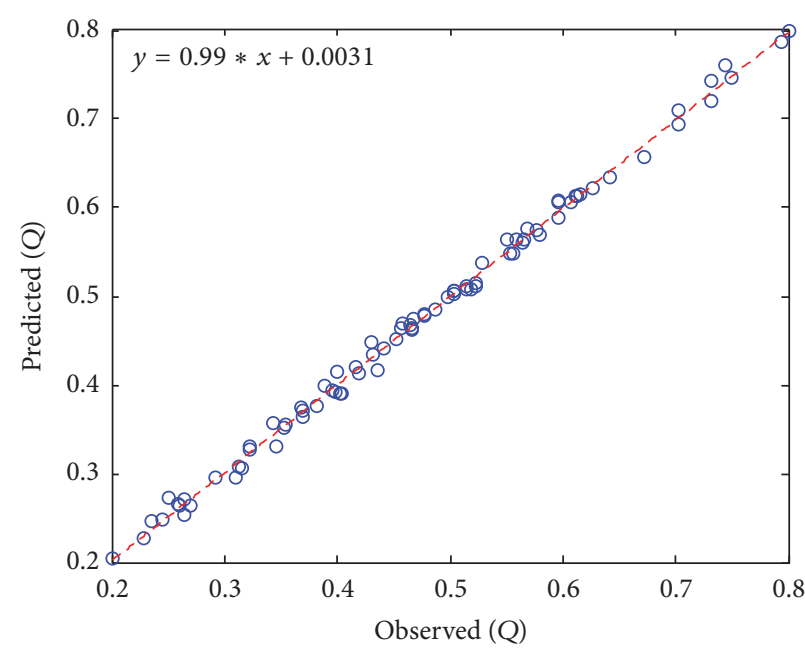

(a)

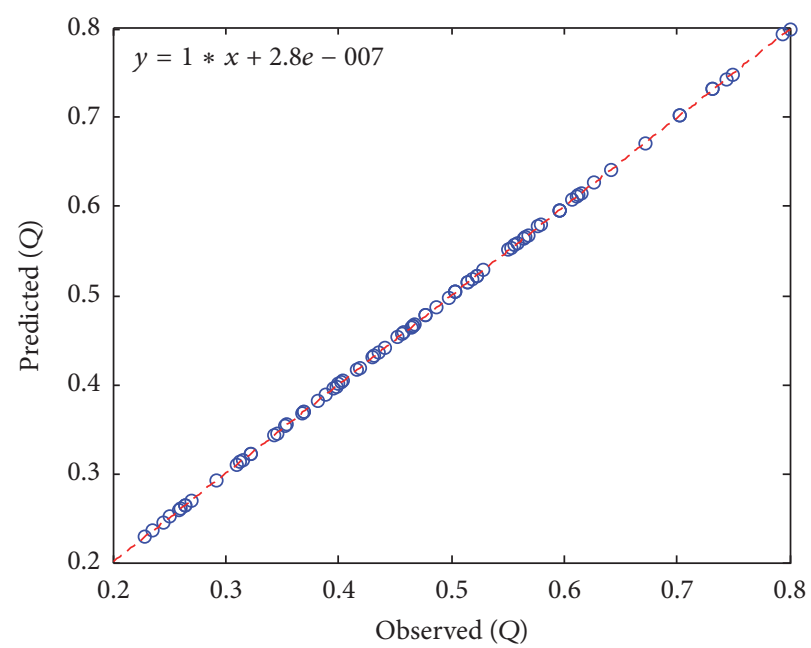

(b)

FIGURE 6: Training performance of the designed models (a) NHW and (b) DANFIS.

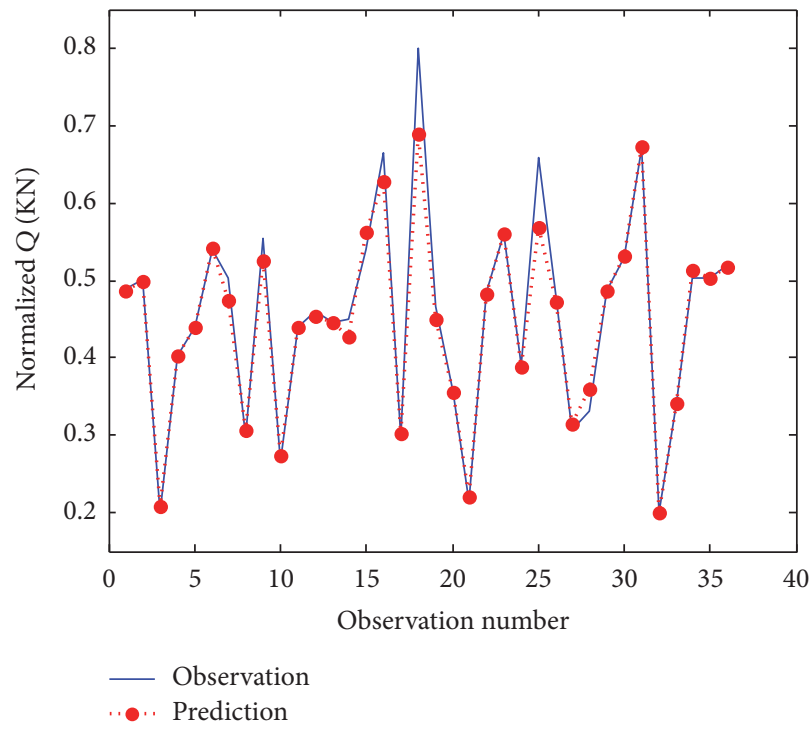

(a)

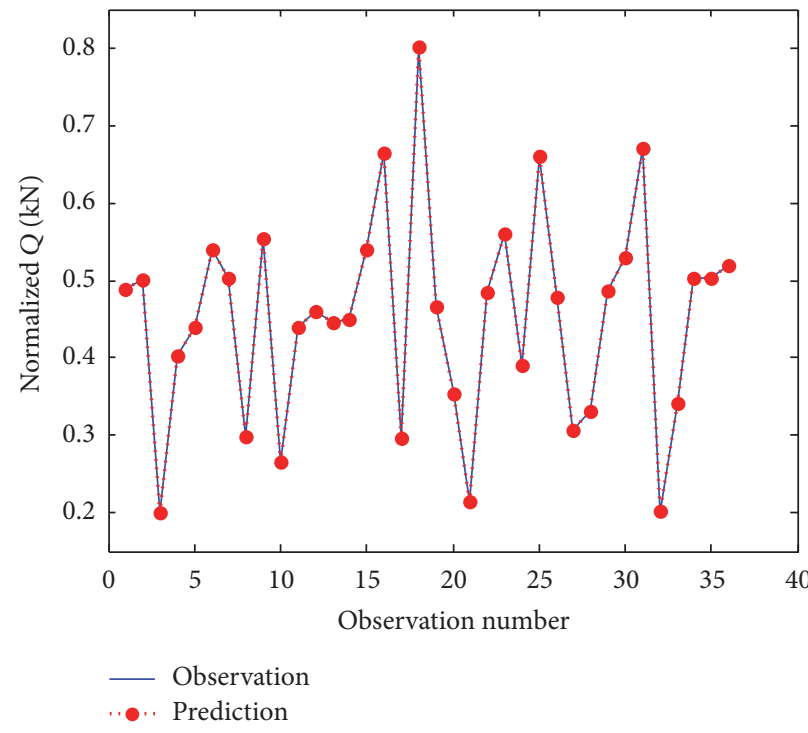

(b)

FIGURE 7: Testing performance of the designed models (a) NHW and (b) DANFIS.

TABLE 6: Comparison between designed models and LSSVM [5] model for the testing data.

\begin{tabular}{lccc}
\hline Model & RMSE $(\mathrm{KN})$ & MAE $(\mathrm{KN})$ & $R$ \\
\hline LSSVM [5] & 0.26 & 0.20 & 0.94 \\
NHW & $2.71 E-2$ & $1.32 E-2$ & 0.98 \\
DANFIS & $6.47 E-4$ & $4.03 E-4$ & 0.99 \\
\hline
\end{tabular}

for the ANN and LSSVM models, respectively [5]. As shown in Table 6 and Figure 7, the developed models predict the pullout capacity for the testing data with less RMSE $(2.71 E-2$ and $6.47 E-4$, for the NHW and DANFIS, resp.) and higher accuracy of $R$ (0.98 and 0.99 , for the NHW and DANFIS, resp.). Accordingly, the performance of the DANFIS model is better than the other models in predicting the pullout capacity of ground anchors.

Finally, the models proposed, DANFIS and NHW, can be used to detect the pullout capacity with high accuracy with the DANFIS performing better than the NHW.

\section{Conclusions}

In this study, two models are developed using nonlinear integrated system, which are nonlinear Hammerstein-Wiener (NHW) and delay inputs for the adaptive neurofuzzy inference system (DANFIS) to predict the pullout capacity of small ground anchors. The input variables sensitivity is studied to evaluate the variables effectiveness in prediction using polynomial regression model. The sensitivity analysis shows 
high effect of the equivalent anchor diameter, embedment depth, average cone resistance along the embedment depth, and average sleeve friction along the embedment depth variables in predicting the pullout capacity. The results of the developed models are evaluated using case study data and compared with previous studies. It is concluded that the two proposed models can be used to predict the pullout capacity with high accuracy. Moreover, the performance of the DANFIS outperforms the NHW model in training and testing dataset.

\section{Conflicts of Interest}

The authors declare that there are no conflicts of interest regarding the publication of this paper

\section{Acknowledgments}

This research was supported by Post-Doctor Research Program in 2017 through the Incheon National University (INU), Incheon, South Korea.

\section{References}

[1] R. S. Merifield and S. W. Sloan, "The ultimate pullout capacity of anchors in frictional soils," Canadian Geotechnical Journal, vol. 43, no. 8, pp. 852-868, 2006.

[2] M. A. Shahin and M. B. Jaksa, "Pullout capacity of small ground anchors by direct cone penetration test methods and neural networks," Canadian Geotechnical Journal, vol. 43, no. 6, pp. 626-637, 2006.

[3] A. M. Fahmy, J. R. de Bruyn, and T. A. Newson, "Numerical investigation of the inclined pullout behavior of anchors embedded in clay," Geotechnical and Geological Engineering, vol. 31, no. 5, pp. 1525-1542, 2013.

[4] Z. A. Ardebili, M. A. Gabr, and M. S. Rahman, "Uplift capacity of plate anchors in saturated clays: analyses with different constitutive models," International Journal of Geomechanics, vol. 16, no. 2, Article ID 4015053, 11 pages, 2016.

[5] P. Samui, D. Kim, and B. G. Aiyer, "Pullout capacity of small ground anchor: a least square support vector machine approach," Journal of Zhejiang University: Science A, vol. 16, no. 4, pp. 295-301, 2015.

[6] M. A. Shahin and M. B. Jaksa, "Neural network prediction of pullout capacity of marquee ground anchors," Computers and Geotechnics, vol. 32, no. 3, pp. 153-163, 2005.

[7] M. Shahin and M. Jaksa, "Modelling the pullout capacity of marquee ground anchor," Research Report R174, School of civil and enviromental Engineering, University of Adeliade, Adelaide, Australia, 2003.

[8] L. Ljung, Q. Zhang, P. Lindskog, and R. Singh, "An integrated system identification tool-box for linear and non-linear models," in Proceedings of the 14th IFAC Symposium on Identification and System Parameter Estimation, pp. 931-936, Newcastle, Australia, 2006.

[9] L. Ljung, "System identification toolbox," Matlab User's Guid, vol. 1, article 237, 2011.

[10] D. Zambrano, S. Tayamon, and B. Carlsson, "Identification of a discrete-time nonlinear Hammerstein-Wiener model for a selective catalytic reduction system," in Proceedings of the American Control Conference (ACC '11), pp. 8-13, June 2011.

[11] M. R. Kaloop, J. W. Hu, and M. A. Sayed, "Bridge performance assessment based on an adaptive neuro-fuzzy inference system with wavelet filter for the GPS measurements," ISPRS International Journal of Geo-Information, vol. 4, no. 4, pp. 2339-2361, 2015.

[12] M. Eldessouki and M. Hassan, "Adaptive neuro-fuzzy system for quantitative evaluation of woven fabrics' pilling resistance," Expert Systems with Applications, vol. 42, no. 4, pp. 2098-2113, 2015.

[13] O. Kisi and J. Shiri, "Prediction of long-term monthly air temperature using geographical inputs," International Journal of Climatology, vol. 34, no. 1, pp. 179-186, 2014.

[14] K. S. Arsava, Y. Kim, T. El-Korchi, and H. S. Park, "Nonlinear system identification of smart structures under high impact loads," Smart Materials and Structures, vol. 22, no. 5, Article ID 055008, 2013.

[15] F. Guo, A new identification method for Wiener and Hammerstein systems [Ph.D. thesis], Institute for Applied Computer Science, Karlsruhe, Germany, 2004.

[16] T. Patikirikorala, L. Wang, A. Colman, and J. Han, "Hammerstein-Wiener nonlinear model based predictive control for relative QoS performance and resource management of software systems," Control Engineering Practice, vol. 20, no. 1, pp. 49-61, 2012.

[17] A. Wills, T. B. Schön, L. Ljung, and B. Ninness, "Identification of Hammerstein-Wiener models," Automatica, vol. 49, no. 1, pp. 70-81, 2013.

[18] J.-S. R. Jang, "ANFIS: adaptive-network-based fuzzy inference system," IEEE Transactions on Systems, Man and Cybernetics, vol. 23, no. 3, pp. 665-685, 1993.

[19] K. S. Arsava, Y. Kim, K. H. Kim, and B.-S. Shin, "Smart fuzzy control of reinforced concrete structures excited by collisiontype forces," Expert Systems with Applications, vol. 42, no. 21, pp. 7929-7941, 2015.

[20] H. Adeli and X. Jiang, "Dynamic fuzzy wavelet neural network model for structural system identification," Journal of Structural Engineering, vol. 132, no. 1, pp. 102-111, 2006.

[21] T. Takagi and M. Sugeno, "Fuzzy identification of systems and its applications to modeling and control," IEEE Transactions on Systems, Man and Cybernetics, vol. 15, no. 1, pp. 116-132, 1985.

[22] M. R. Kaloop, J. W. Hu, and Y. Bigdeli, "Identification of the response of a controlled building structure subjected to seismic load by using nonlinear system models," Applied Sciences, vol. 6, no. 10, p. 301, 2016.

[23] I. Mansouri, A. Gholampour, O. Kisi, and T. Ozbakkaloglu, "Evaluation of peak and residual conditions of actively confined concrete using neuro-fuzzy and neural computing techniques," Neural Computing and Applications, pp. 1-16, 2016.

[24] H. Erdogan and E. Gulal, "The application of time series analysis to describe the dynamic movements of suspension bridges," Nonlinear Analysis. Real World Applications, vol. 10, no. 2, pp. 910-927, 2009.

[25] M. R. Kaloop, J. W. Hu, and E. Elbeltagi, “Time-series and frequency-spectrum correlation analysis of bridge performance based on a real-time strain monitoring system," ISPRS International Journal of Geo-Information, vol. 5, no. 5, article 61, 2016.

[26] H. Erdogan and E. Gülal, "Identification of dynamic systems using multiple input-single output (MISO) models," Nonlinear Analysis. Real World Applications, vol. 10, no. 2, pp. 1183-1196, 2009. 
[27] M. Bustamente and L. Gianeselli, "Pile bearing capacity prediction by means of static penetrometer CPT," in Proceedings of the 2nd European Symposium on Penetration Testing, pp. 493-500, 1982.

[28] B. M. Das, Principles of Foundation Engineering, Cengage Learning, Boston, Mass, USA, 7th edition, 2010.

[29] J. E. Bowles, Foundation Analysis, McGraw-Hill, New York, NY, USA, 3rd edition, 2001.

[30] P. Samui and T. G. Sitharam, "Pullout capacity of small ground anchors: a relevance vector machine approach," Geomechanics and Engineering, vol. 1, no. 3, pp. 259-262, 2009. 


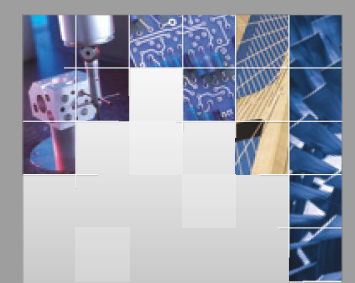

\section{Enfincering}
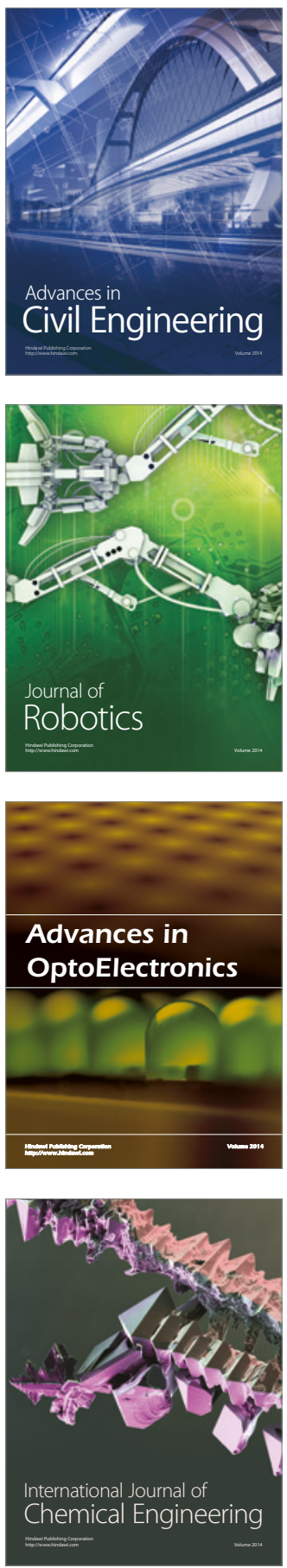

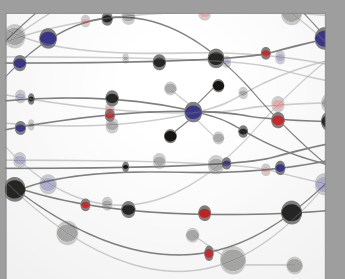

The Scientific World Journal

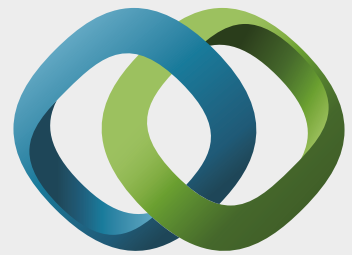

\section{Hindawi}

Submit your manuscripts at

https://www.hindawi.com
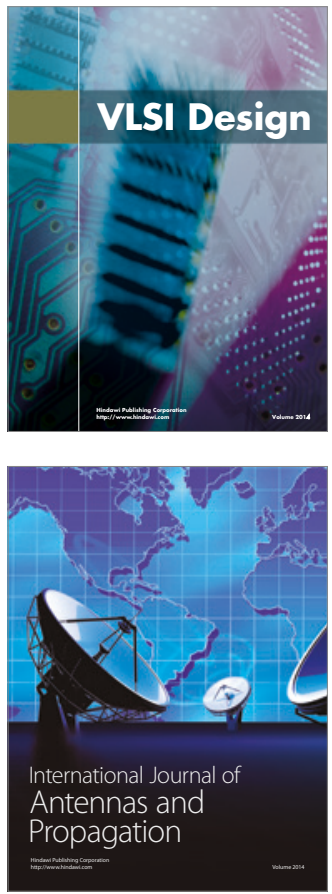

\section{Rotating}

Machinery
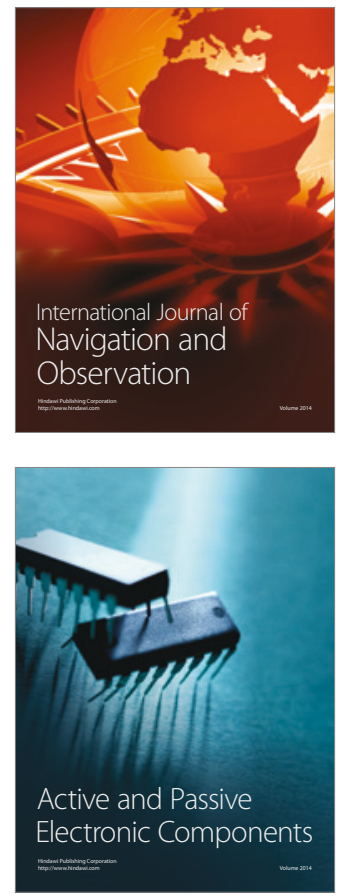
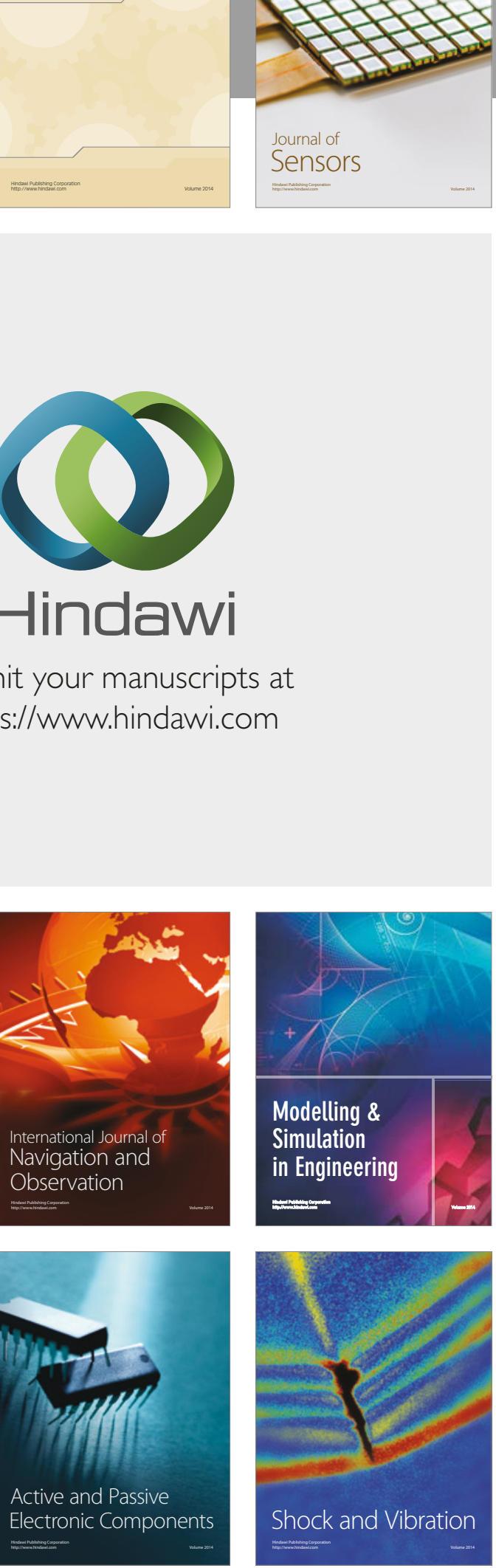
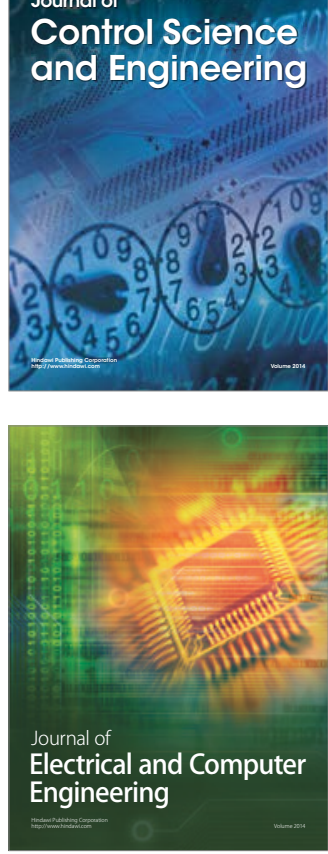

Distributed

Journal of

Control Science

and Engineering
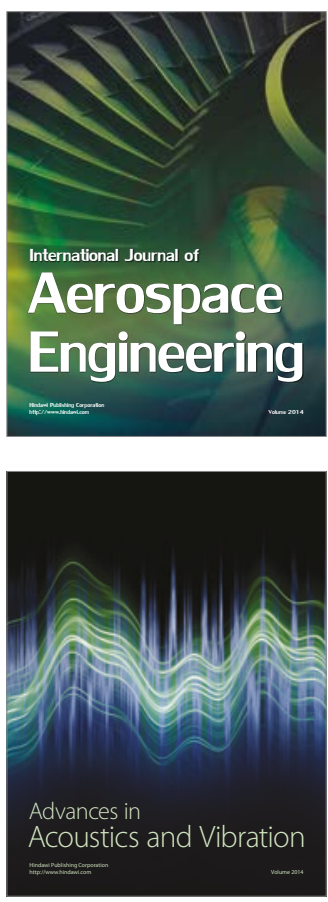

Sensor Networks 